\title{
Descriptive study of oral health, dental care and health behavior of inpatients undergoing physical medicine and rehabilitation
}

\author{
Júlia Moldvai $^{{ }^{*} \text {, Mercédesz Orsós }}{ }^{1}$, Fanni Simon ${ }^{1}$, Gergő $_{\text {Merész }}{ }^{2}$ and Orsolya Németh ${ }^{1}$ \\ ${ }^{1}$ Department of Community Dentistry, Semmelweis University, Budapest, Hungary \\ ${ }^{2}$ Doctoral School of Mental Health Sciences, Semmelweis University, Budapest, Hungary
}

\begin{abstract}
Background: Regarding to the WHO's World report on disability, more than 1 billion people are estimated to live with some form of disability. According to the 2011 census in Hungary their number can be around 500.000. These people are considered as a vulnerable group even from a dental point of view, since the loss of function makes regular oral hygienic routine and dentistry control more difficult.
\end{abstract}

Aim: In 2015 The Department of Community Dentistry and The National Institute of Medical Rehabilitation started to operate together a dental office. We gathered comprehensive information on patient's oral health currently undergoing rehabilitation and living with disabilities.

Method: This study was approved by the Medical Research Council, Hungary. The clinical examination were done by the recommendation of WHO. A total of 608 inpatients have gone through a full dental examination including a stomato-oncological screening. We analyzed the DMF-T values. Oral hygiene routine, dental care, demographic characteristics, self-reported smoking, drinking and eating habits related median DMF-T were surveyed.

Results: The patients had poor oral hygiene. The median DMF-T was 24 in age group'55 years or older'. Their complaints about dental and oral mucosal lesions are minimal compared to their general dental condition. $28.91 \%$ of the inpatients reported toothache in the past 12 months, whose median DMF-T was 19 . The last dental checkup in the case of most patients exceeds 24 months.

Conclusion: All of the values are worse than the average Hungarian population. Their oral hygiene is poor since the daily oral hygiene routine and care in practice is not solved for these patients.

\section{Introduction}

Regarding to the WHO's World report on disability, more than 1 billion people are estimated to live with some form of disability [1]. According to the 2011 census in Hungary their number can be around $500.000,4.6 \%$ of the society [2]. The number is increasing continuously due to ever-ageing populations and the high number of chronical diseases like diabetes, cardiovascular and mental problems [3]. Due to the consequences of these diseases or injury caused by accident have lost their autonomy in whole or in part. Depending on the severity of their condition, they have varying degrees of disadvantage in maintaining their daily routine. These people with special health care needs are considered as a vulnerable and underserved group even from a dental point of view, since the loss of function makes regular oral hygienic routine and dentistry control more difficult [4]. It is also complicated to reach the dental office or even if they can reach it, they often do not receive appropriate treatment because dentists are afraid of treating patients requiring special care or do not have enough experience in this field. Availability of dental providers trained to serve special needs populations are limited $[5,6]$.

There are no policies and protocols in place regarding prevention of such patients because there is no available data in Hungary on the oral hygiene or dental status of patients undergoing rehabilitation. Physical medicine and rehabilitation is an interdisciplinary teamwork and dental professionals have to be a part of it. Oral health care and oral rehabilitation have to be components of rehabilitation [7].

In 2015 The Department of Community Dentistry, Semmelweis University and The National Institute of Medical Rehabilitation started to operate together a dental office to gain comprehensive information on patient's oral health, health behaviour and dental care undergoing rehabilitation. We have also been informed about oral hygiene practices, the most consumed nutriments, addictive substances. Sociodemographic data such as age, gender and inhibitancy were collected. Clinical data on the reason for rehabilitation, the underlying diseases were derived from patients' files.

\section{Materials and methods}

This study was conducted in Hungary at The National Institute of Medical Rehabilitation. A total of 608 inpatients have gone through a

${ }^{\star}$ Correspondence to: Júlia Moldvai DMD, Department of Community Dentistry, Semmelweis University, Budapest, Hungary; E-mail: moldvai.julia@ dent.semmelweis-univ.hu

Key words: disability, health behavior, rehabilitation, oral hygiene, epidemiology

Received: January 21, 2019; Accepted: January 30, 2019; Published: February 01, 2019 
full dental examination between $5^{\text {th }}$ of April 2016 and $20^{\text {th }}$ of January 2018. The consent of the appropriate ethical committee (Medical Research Council, Hungary, ETT TUKEB 4913 /2016/ EKU) was acquired prior to start of this study. The participants have provided written consent. In case of infants, the parents/legal guardians have given consent.

The examinations were carried out by two calibrated dentists. The standardized dental examination were performed according to WHO standards. The clinical examination and the questionnaire for the health behaviour and dental care were done by recommendation of WHO. The instruments for oral examination to gain information on oral health were plane mouth mirror and dental probe using artificial light. From a survey of 20 questions we asked about oral hygiene practices, toothbrushing frequency, last visit to a dentist, eating habits, addictions and social background.

The patients were examined for dental caries, missing and filled tooth (sound crown; decayed crown; filled crown with decay; filled crown, with no decay; missing tooth). Diagnostical codes and criteria for recording the dentition status were according to the World Health Organization [8].

Demographic variables consisted of gender, age and permanent address. Dental care and oral health was measured with few important questions like frequency of toothbrushing, last dental visit, using adjuncts, toothache in last one year, wearing dentures, and using denture cleaner. Demographic characteristics, dental care, self-reported smoking, drinking and eating habits related median DMF-T were surveyed.

Responses were collected on Android tablets. The software and the application was created by a collaboration between us and the scientists of Wigner Research Centre for Physics and the data was uploaded directly to the cloud service of Hungarian Academy of Science.

Statistical analysis were performed using R software. The validity of data entry was checked by randomly selecting observations and checking their entries against the original questionnaires. The descriptive data are presented as frequency, mean, (standard deviation (SD)) and median.

\section{Results}

The dental screening was at nine departments of The National Institute of Medical Rehabilitation: Department of Orthopedics (72), Surgical and Rehabilitation Department of Amputations (9), Rehabilitation Department of Traumatic Injuries (60), Rehabilitation Department of Spinal Cord Injuries (87), Rehabilitation Department of Hemiplegics (91), Rehabilitation Department of Brain Injuries
(87), Department of Bone and Joint Infection and TBC Rehabilitation (88), Rehabilitation Ward of patients with different disabilities (101), Department of Psychosomatic and Psychotherapeutic Rehabilitation (13).

Distribution of DMF-T scores according to demographic related factors is shown in Table 1. There were 311 (51.15\%) male and 297 (48.85\%) female patients, whose median DMF-T scores were 19 and 21. $72 \%$ of the participants were older than 55 years old and their DMF-T scores were the highest.

$172(28.91 \%)$ patients reported toothache in the past 12 months, whose median DMF-T scores was 19 . The last dental checkup in the case of most patients exceeds 24 months. There were 202 patients whose last dental visit was more than two years ago instead of the recommended half year. The relationship between the last dental screening and DMF-T scores was visible. 345 (57.69\%) patients performed tooth brushing more than twice or more per day but $68(11.37 \%)$ participants of the cohort cleaned their teeth less than daily. The majority of the patients $(87.62 \%)$ used manual toothbrush. Patients who used some kind of adjuncts for example dental floss for tooth cleansing had got better DMF-T scores. 211 patients wear removeable denture (Table 2). Among 108 (17.76\%) patients partial and 103 (16.94\%) complete dentures used, but not all of these patients reported use of denture cleaner (Table 3 ).

Distribution of median DMF-T scores according to self-reported smoking, drinking and eating habits is shown in Table 4.118 (19.47\%) patients smoked. There is no apparent difference between the values of smoking and non-smoking patients. 471 (82.63\%) participants did not drink alcohol in the past 30 days. The relationship between the foods that are at risk and the value of DMF-T was noticeable. 223 (36.68\%) people who ate less than 2 types of high risk foods had got the best DMF-T scores.

\section{Discussion}

DMF-T score is a good indicator of populations' oral health. Looking at the DMF-T distribution by age group, we can say that those above 55 have double the value than those who are 44 or younger, which can be explained by loss of teeth. If we look at the DMF-T value for the average Hungarian population, one can see that the results are significantly higher [9].

In the last 12 months, $69.41 \%$ of the patients did not report toothaches which is contradict to their oral condition. This can be explained by the fact that this group of patients get several painkillers during their rehabilitation which as a second effect hides their oral complaints [10].

Table 1. Demographic characteristics ( $\mathrm{n}=608$ for the total cohort; data reported as frequency, mean (SD) and median)

\begin{tabular}{|c|c|c|c|}
\hline Characteristic & n (\%) & Mean DMF-T (SD) & Median DMF-T \\
\hline \multicolumn{4}{|l|}{ Gender } \\
\hline Male & $311(51.15 \%)$ & $18.86(7.60)$ & 19 \\
\hline Female & $297(48.85 \%)$ & $19.88(7.29)$ & 21 \\
\hline \multicolumn{4}{|c|}{ Age group $(n=606)$} \\
\hline$\leq 44$ years & $132(21.78 \%)$ & $12.20(6.76)$ & 12 \\
\hline 45-54 years & $100(16.50 \%)$ & $16.58(5.27)$ & 16 \\
\hline$\geq 55$ years & $374(61.72 \%)$ & $22.56(6.09)$ & 24 \\
\hline \multicolumn{4}{|c|}{ Permanent address $(n=608)$} \\
\hline Capital & $260(42.76 \%)$ & $20.32(6.90)$ & 21 \\
\hline Other & $348(57.24 \%)$ & $18.64(7.79)$ & 19 \\
\hline
\end{tabular}


Table 2. Oral health of the cohort ( $\mathrm{n}=608$ for the total cohort; data reported as frequency, mean (SD) and median)

\begin{tabular}{|c|c|c|c|}
\hline Indicator & n (\%) & Mean (SD) & Median DMF-T \\
\hline \multicolumn{4}{|l|}{ Toothache in the past 12 months $(n=596)$} \\
\hline Reported & $172(28.91 \%)$ & $18.46(6.91)$ & 19 \\
\hline Not Reported & $413(69.41 \%)$ & $19.67(7.70)$ & 21 \\
\hline Don't know & $11(1.85 \%)$ & $20.00(7.29)$ & 19.5 \\
\hline \multicolumn{4}{|l|}{ Last dental visit $(\mathrm{n}=598)$} \\
\hline$\leq 6$ months ago & $173(28.93 \%)$ & $19.68(6.90)$ & 20 \\
\hline 6-12 months ago & $134(22.41 \%)$ & $18.21(7.60)$ & 19 \\
\hline 12-24 months ago & $89(14.88 \%)$ & $17.77(7.87)$ & 18.5 \\
\hline$\geq 24$ months ago & $202(33.78 \%)$ & $20.58(7.56)$ & 22 \\
\hline \multicolumn{4}{|l|}{ Self-reported frequency of tooth brushing $(n=598)$} \\
\hline Twice or more per day & $345(57.69 \%)$ & $18.83(7.46)$ & 19 \\
\hline Daily & $185(30.94 \%)$ & $19.48(7.70)$ & 20 \\
\hline Less than daily & $68(11.37 \%)$ & $20.87(6.49)$ & 21.5 \\
\hline \multicolumn{4}{|l|}{ Adjuncts used for tooth cleansing $(n=606)$} \\
\hline Toothbrush (electric) & $114(18.81 \%)$ & $16.23(6.98)$ & 16 \\
\hline Toothbrush (manual) & $531(87.62 \%)$ & $19.32(7.45)$ & 20 \\
\hline Toothbrush (manual or electric) and mouthwash & $300(49.50 \%)$ & $19.25(7.07)$ & 19 \\
\hline Toothbrush (manual or electric), mouthwash and dental floss or shiwak or other & $51(8.42 \%)$ & $17.16(6.92)$ & 17 \\
\hline \multicolumn{4}{|l|}{ Dentures $(n=608)$} \\
\hline Not reported & $397(65.30 \%)$ & $16.56(6.99)$ & 16 \\
\hline Partial & $108(17.76 \%)$ & $25.05(3.64)$ & 26 \\
\hline Complete & $103(16.94 \%)$ & $27.09(2.07)$ & 28 \\
\hline Partial and complete & $30(4.93 \%)$ & $27(1.82)$ & 28 \\
\hline
\end{tabular}

Table 3. Self-reported use of denture cleaner by type of dentures $(\mathrm{n}=181)^{*}$

\begin{tabular}{|l|c|c|c|c|}
\hline \multicolumn{2}{|c|}{} & \multicolumn{3}{|c|}{ Type of dentures } \\
\cline { 2 - 5 } & & Partial only & Complete only & Partial and complete \\
\hline \multirow{3}{*}{ Use of denture cleaner } & Not reported & 44 & 23 & 10 \\
\cline { 2 - 5 } & Reported & 34 & 50 & 20 \\
\cline { 2 - 5 } & Total & 78 & 73 & 30 \\
\hline
\end{tabular}

"The reported use of dental prosthesis cleaner was significantly different between groups of patients according to the type of dental prosthesis $\left(\mathrm{X}^{2}=10.815, \mathrm{p}=0.004\right)$

Table 4. Self-reported smoking, drinking and eating habits ( $\mathrm{n}=608$ for the total cohort; data reported as frequency, mean (SD) and median)

\begin{tabular}{|c|c|c|c|}
\hline Characteristic & n (\%) & Mean DMF-T (SD) & Median DMF-T \\
\hline \multicolumn{4}{|l|}{ Smoking $^{1}(n=606)$} \\
\hline Yes & $118(19.47 \%)$ & $18.75(7.42)$ & 19 \\
\hline No & $448(80.52 \%)$ & $19.51(7.48)$ & 20 \\
\hline \multicolumn{4}{|c|}{ Alcohol consumption $^{2}(\mathrm{n}=570)$} \\
\hline Any other reply & $99(17.37 \%)$ & $18.11(7.62)$ & 18 \\
\hline Not in the past 30 days & $471(82.63 \%)$ & $19.57(7.47)$ & 20 \\
\hline \multicolumn{4}{|c|}{ Daily consumption of high risk food types ${ }^{3}$} \\
\hline$\geq 6$ & $40(6.58 \%)$ & $19.13(8.30)$ & 20.5 \\
\hline 5 & $64(10.53 \%)$ & $20.66(7.12)$ & 22.5 \\
\hline 4 & $122(20.06 \%)$ & $20.15(7.14)$ & 21 \\
\hline 3 & $159(26.15 \%)$ & $20.03(7.30)$ & 21 \\
\hline$\leq 2$ & $223(36.68 \%)$ & $18.09(7.58)$ & 18 \\
\hline
\end{tabular}

${ }^{1}$ Subcategories of smoking are defined by aggregating the number of patients who reported either the permanent or occasional consumption of any tobacco product (cigarettes, cigars, pipe, snuff or any other kind)

${ }^{2}$ Subcategories of alcohol consumption are created by aggregating the number of patients who reported to regularly consume any amount of alcohol in the past 30 days.

${ }^{3}$ Subcategories of high risk food types are fresh fruit, pies and buns, jam and honey, chewing gum containing sugar, sugar or any kind of sweetener, lemonade or other soft drinks, tea served with sugar and coffee served with sugar.

There is strong correlation between the last dental screening and the DMF-T value. Only $28.93 \%$ of patients go to screening every 6 months, this is due to systematic barriers to oral health care such as transportation and cost. A US survey suggests that the dental care for special-need patients is not sufficient and lack of access to oral health care contributes oral health disparities [5,4].

$57.69 \%$ of patients clean their teeth according to the recommended two times daily. There is no protocol in Hungary for preventing dental disease in patients undergoing rehabilitation. Such protocols and checkups are often overlooked within hospitals. Oral health care of hospitalized patients is often neglected due to the priority of other medical care [11]. Many of these patients suffer deficiencies due to a deterioration of manual function; teeth cleaning is more difficult where they can not seize their manual toothbrush. The electric toothbrush can be a good solution due to their better plaque removal effectiveness [12]. In our study the use of electric toothbrush shows better DMF-T value, however only a small 
proportion of patients use it for proper dental hygiene. The use of dental supplements such as dental floss, taking into account the loss of physical functioning can be difficult. The electric toothbrush is not common and due to its high price often cannot be purchased in this population [13].

More than one third of inpatients have some kind of removable denture. However $42.54 \%$ do not use denture cleaner and many do not know that such use is necessary, which shows also a lack of prevention [14].

High percentage of inpatients have given up addictions and do not consume alcohol or tobacco. $80.52 \%$ of patients undergoing rehabilitation do not smoke and $82.63 \%$ did not drink alcohol in the past 30 days. This can be explained by the difficulty of acquiring such substances during rehabilitation.

Out of the many high-risk foods, we can observe that the more patients consume them, the worse their DMF-T value becomes. This is explained by the high risk of prevalence of caries when consuming fermentable carbohydrates [15].

\section{Conclusion}

Our study demonstrated the correspondence between the health behavior and the DMF-T values. The results of our research points to the fact that people undergoing rehabilitation have difficulties in developing and maintaining proper oral health due to their disadvantages and therefore require special care and attention. With the help of our results, we can create new dental prevention models that help adapt patients with special needs to their changed circumstances. Thus, restoring their autonomy to patients can provide an opportunity for reintegration into society.

\section{Conflict of interest}

The authors declare no conflict of interest.

\section{References}

1. World Health Organization (2011) World report on disability.

2. Hungarian Central Statistical Office (2011) Population Census, Disabled persons.

3. WHO Fact sheets: Disability and health. https://www.who.int/news-room/fact-sheets/ detail/disability-and-health

4. IOM (2011) (Institute of Medicine) and NRC (National Research Council): Improving access to oral health care for vulnerable and underserved populations. Washington, DC: The National Academies Press.

5. U.S. Department of Health and Human Services (2000) Oral health in America: report of the Surgeon General. Rockville (MD): U.S. Department of Health and Human Services; National Institute of Dental and Craniofacial Research; National Institutes of Health.

6. Ship JA, Chavez EM (2000) Management of systemic diseases and chronic impairment in older adults: oral health considerations. Gen Dent pp. 555-565. [Crossref]

7. Waldman HB, Perlman SP (2010) Disability and rehabilitation: do we ever think about needed dental care? A case study: the USA. Disabil Rehabil 32: 947-951. [Crossref]

8. World Health Organization (2013) Oral health surveys: basic methods - (5th Edn) WHO.

9. Madléna M, Hermann P, Jáhn M, Fejérdy P (2008) Caries prevalence and tooth loss in Hungarian adult population: results of a national survey. BMC Public Health 8: 364. [Crossref]

10. Orsós M, Moldvai J, Kivovics P, Németh O (2018) Oral health related quality of life of patients undergoing physical medicine and rehabilitation. Orv Hetil 159: 2202-2206.

11. Zhu HW, McGrath C, McMillan AS, Li LS (2008) Can caregivers be used in assessing oral health-related quality of life among patients hospitalized for acute medical conditions? Community Dent Oral Epidemiol 36: 27-33. [Crossref]

12. Bartizek RD, Biesbrock AR (2002) Dental plaque removal efficacy of a batterypowered toothbrush vs. a control Japanese manual toothbrush. Am J Dent $15 \mathrm{Spec}$ No: 33A-36A. [Crossref]

13. Eurostat (2015) Disability statistics - poverty and income inequalities. Data from August 2015.

14. Glassman P, Anderson M, Jacobsen P, Schonfeld S, Weintraub J, et al. (2003) Practical protocols for the prevention of dental disease in community settings for people with special needs: the protocols. Spec Care Dentist 23: 160-164.

15. Hujoel PP, Lingström P (2017) Nutrition, dental caries and periodontal disease: a narrative review. J Clin Periodontol 18: S79-S84. [Crossref]

Copyright: (C2019 Moldvai J. This is an open-access article distributed under the terms of the Creative Commons Attribution License, which permits unrestricted use, distribution, and reproduction in any medium, provided the original author and source are credited. 